\title{
Défis et avancées de la construction territoriale régionale en Outaouais : le cas forestier
}

Edith Leclerc

Université d'Ottawa

\section{INTRODUCTION}

Reconnue comme une mesure décentralisatrice, la mise en place de Commissions régionales sur les ressources naturelles et le territoire public (CRRNT), autour des années 2010, résulte d'un processus entamé plusieurs années auparavant. Déjà, dans son rapport de 2004, la Commission d'étude sur la gestion de la forêt publique québécoise, mieux connue sous le nom de Commission Coulombe, propose cinq virages à entreprendre pour l'avenir forestier québécois. Parmi les autres, le virage qui retient notre attention ici est celui de décentraliser la gestion forestière afin de réaliser des objectifs de transparence, d'information et de participation ${ }^{1}$.

\section{L'Outaouais ne fait pas exception à cette demande d'un plus grand pouvoir régional à définir les orientations forestières et d'une meilleure distribution du financement forestier.}

Or, bien avant cette proposition de la Commission Coulombe, les instances municipales et régionales voyaient dans l'aménagement des ressources naturelles à la fois une compétence nécessaire afin de réaliser pleinement leur mandat d'aménagement du territoire, à la fois comme un moyen de mettre en valeur leur potentiel de développement territorial. Longtemps exclues du circuit décisionnel autour des ressources naturelles, les régions et les municipalités subissent pourtant les contre coups des décisions en matière de politiques forestières ainsi que des choix industriels face à l'aménagement ${ }^{2}$. Lors des audiences de la Commission Coulombe les instances régionales et municipales, telles que les Conférences régionales des élus (CRÉ), les municipalités régionales de comtés (MRC) et les municipalités, ont été fermes: les ressources naturelles doivent permettre aux régions de devenir des acteurs privilégiés de leur propre développement. L'Outaouais ne fait pas exception à cette demande d'un plus grand pouvoir régional à définir les orientations forestières et d'une meilleure distribution du financement forestier.

Bien avant la Commission Coulombe, les instances municipales et régionales voyaient dans l'aménagement des ressources naturelles une compétence nécessaire afin de réaliser pleinement à la fois leur mandat d'aménagement du territoire, à la fois leur potentiel de développement territorial.

Peu de temps après la publication du rapport de la Commission Coulombe apparaissent, d'abord, les comités régionaux forestiers (approximativement entre 2007 et 2009) qui deviennent, avec la Loi sur l'aménagement durable des territoires forestier, les Commissions régionales sur les ressources naturelles et le territoire public. La nouvelle loi, sanctionnée en 2010, n'entre en vigueur qu'en 2013; ce délai concède aux CRÉ de chacune des régions administratives une marge de manœuvre relative afin de se constituer et/ou consolider les CRRNT qui doivent, dans cette même période, réaliser une première planification des objectifs forestiers régionaux (le plan régional de développement intégré des ressources naturelles et du territoire - PRDIRT).

C'est à cette mise en place et au processus d'élaboration de ce premier PRDIRT que notre analyse s'est intéressée. Notre texte rend compte des avancées et des défis réalisés en Outaouais dans la dynamique de construction et d'organisation territoriale autour de la question forestière. 


\section{GENÈSE DE LA TERRITORIALISATION DANS LE SECTEUR FORESTIER QUÉBÉCOIS}

L'apparition d'instances décentralisées dans le secteur forestier ne découle pas systématiquement et directement de la Loi sur l'aménagement durable des forêts de 2010. Déjà, avec la Loi sur les forêts de 1986 et ses modifications subséquentes, des mécanismes décentralisés se manifestaient. Les premières formes de décentralisation étaient pilotées par les industries forestières, qui devaient soumettre leurs planifications forestières aux usagers des territoires qui avaient d'autres activités que la récolte fibreuse (chasseurs/ trappeurs ou pêcheurs, randonneurs de tous types). Cette décentralisation visait d'abord à informer ces autres utilisateurs du territoire, puis, plus tard, à les consulter. Cette distinction apparait importante en sémantique, mais les usagers, eux, n'y ont pas nécessairement distingué les nuances qui sont à faire entre information et consultation ${ }^{3}$. Devant l'insatisfaction des utilisateurs du territoire forestier à l'égard de ces processus et de leurs retombées concrètes, les modifications législatives de 2001 à la Loi sur les forêts sont venues encadrer les processus et conditions de la participation de la gestion forestière, en particulier dans les instances décentralisées. Cette territorialisation, comprise comme le rapprochement des centres décisionnels vers les acteurs locaux et régionaux, a mis au jour des processus de concertation et d'intégration de cette concertation dans la gestion des ressources forestières.

Une étude portant sur les tables locales de gestion intégrée montre la signification de la mise en place de telles tables locales ${ }^{4}$. Dans une perspective favorable, les tables locales deviennent des lieux de rencontre. Elles permettent de prendre conscience de l'autre, de sa place et de ses besoins. En somme, elles permettent de reconnaître l'autre et de traiter ensemble des problématiques vécues, autant des siennes que de celles des autres. Du revers, l'étude a montré l'importance d'un pilotage neutre, ce qui n'était pas le cas pour toutes les tables locales mises en place alors. Aussi, l'analyse des deux cas étudiés fait la preuve de l'effort considérable à mettre dans la conver- gence des acteurs vers les lieux communs, tant physiquement - par exemple à rassembler l'ensemble des acteurs territoriaux aux rencontres - qu'idéologiquement - c'est-à-dire à avoir une discussion fluide plutôt que des confrontations.

L'organisation territoriale dite « en millefeuilles » montre la complexité de l'organisation de la gouvernance forestière au Québec de l'échelle locale à l'échelle provinciale.

Le modèle des tables locales de gestion intégrées a été revalorisé par la loi sur l'aménagement durable des territoires forestiers, les plaçant sous l'autorité de nouvelles instances régionales; les Commissions régionales sur les ressources naturelles et le territoire (CRRNT), elles-mêmes dépendantes des Conseils régionaux des élus. Cette organisation territoriale dite « en mille-feuilles » montre la complexité de l'organisation de la gouvernance forestière au Québec de l'échelle locale à l'échelle provinciale. L'importance que prend l'administration régionale est alors nouvelle et force est d'admettre l'importance de saisir les tenants et les aboutissants de la création d'une 'nouvelle échelle régionale' dans la gouvernance forestière. Les résultats relatifs à la mise en place de la CRRNT en Outaouais, issus d'une recherche plus large portant sur deux $\mathrm{cas}^{5}$, permettent de discuter des tenants et aboutissants de la création de l'échelle régionale en Outaouais. Au cours de cette recherche, nous avons rencontré 12 intervenants; 10 commissaires siégeant à la CRRNT, un employé ainsi qu'un acteur non membre de la CRRNT. Leurs propos sont rapportés entre «...», sans autres indications.

Le modèle des tables locales de gestion intégrées a été revalorisé par la loi sur l'aménagement durable des territoires forestiers, les plaçant sous l'autorité de nouvelles instances régionales. 


\section{LA MISE EN PLACE DE LA CRRNT EN OUTAOUAIS}

De nombreuses caractéristiques font de l'Outaouais une région intéressante du point de vue de la gouvernance forestière. Parmi d'autres, notons d'abord l'importance historique de la forêt comme moteur économique pour la région, puis insistons sur le caractère unique de l'écosystème forestier de l'Outaouais sur le territoire québécois ${ }^{6}$. Le premier PRDIRT de l'Outaouais a largement été nourri par ces deux caractéristiques qui ont également animé les discussions au sein de la CRRNT. Toutefois, notre réflexion aborde les processus en amont de ces discussions, alors que la CRRNT se mettait en place. Il s'agit ici d'observer les mécanismes, les apprentissages et les obstacles qui émanent de la mise en place de la CRRNT en Outaouais.

Le premier élément observable à la CRRNT est la grande capacité politique de l'organisation. C'est-à-dire que les choix et orientations déterminés par la CRRNT circulent de manière relativement fluide entre les instances, du local au supralocal (MRC) jusqu'aux instances régionales, voire celles provinciales. Un exemple de cela a été la demande régionale d'obtenir un des six bureaux de mise en marché du bois (BMMB) proposés par le gouvernement québécois ${ }^{7}$. La politisation de la CRRNT s'explique par la forte participation des élus municipaux qui composent près de la moitié des commissaires. De l'aveu de la permanence de la CRRNT, cette forte participation des élus ne s'est pas faite d'elle-même et les administrateurs ont dû consentir à beaucoup d'effort pour intégrer et stabiliser la participation des élus. Pour ceux-ci, il est « normal que les élus s'approprient les mécanismes décentralisés puisque la décentralisation passe par les instances politiques locales ». Cela a pour avantage de consolider la relation entre les instances politiques. Du revers, cela rend les discussions à la CRRNT plus politisées; les autres intervenants

\section{CONCLUSION}

En bref, l'avantage de la mise en place d'une structure territoriale autour de la question forestière, comme la CRRNT, est de permettre à chaque territoire régional d'adapter les orientations forestières à ses propres réalités. Si la possibilité d'intégrer ces choix à l'aménagement forestier ont expliqué devoir «marcher sur des œufs afin de ne pas froisser les susceptibilités ».

Notre analyse relève aussi une particularité de fonctionnement de la CRRNT en Outaouais. Il s'agit de la grande capacité à administrer les thématiques de discussion et des projets particuliers à adopter par la CRRNT. Préalablement aux rencontres des commissaires, les dossiers étaient préalablement soumis au souscomité d'aménagement de la CRRNT, composé d'aménagistes et d'experts forestiers. Ce comité avait pour tâche de préparer les dossiers soumis (ou non) aux commissaires et de fixer les informations nécessaires aux points à l'ordre du jour de la CRRNT. Si ce fonctionnement accélère le processus de discussion et allège l'ordre du jour des rencontres (que plusieurs trouvent d'ailleurs très chargé), il biaise les discussions en orientant les thèmes vers des avenues trop communes aux dires de certains.

Enfin, dans le processus d'élaboration du premier PRDIRT de l'Outaouais, notre analyse retient la difficile convergence des acteurs vers un même projet unificateur pour les territoires forestiers de la région. À ce titre, nombreux sont ceux qui considèrent le PRDIRT comme une liste d'épicerie, regroupant ce qui serait souhaitable pour les acteurs forestiers de la région, sans égard à la situation actuelle du secteur forestier et des avenues alternatives possibles. Une explication de cela réside dans le grand contrôle du comité expert sur les orientations de la CRRNT, d'autres l'expliquent par l'urgence dans laquelle la CRRNT s'est retrouvée afin de réaliser le PRDIRT. Cette urgence découle du temps nécessaire à la mise sur pied de la CRRNT, ce qui aurait retardé le début réel des travaux de discussion sur le PRDIRT.

entraine toutefois une complexification du processus de calcul de la possibilité, de l'aménagement et de l'organisation forestière, elle permet de mieux arrimer ces derniers aux orientations d'aménagement du territoire de l'échelle régionale. 


\section{L'avantage de la mise en place d'une structure territoriale autour de la question forestière, comme la CRRNT, est de permettre à chaque territoire régional d'adapter les orientations forestières à ses propres réalités.}

Ainsi, à quoi sert l'échelle régionale? L'exemple de la CRRNT de l'Outaouais montre que la construction territoriale, du moins dans le cas forestier, met de l'avant la dimension sociopolitique du développement et de l'innovation territoriale. Cet aspect se caractérise, par exemple, par une plus grande adaptation des mécanismes décisionnels aux particularités politiques, sociales, économiques et naturelles de chacune des régions.

La déstructuration récente de cette instance a des implications directes pour le milieu forestier de l'Outaouais. Le simple fait de perdre le lieu de convergence des acteurs forestiers afin qu'ils discutent des activités de l'un ou de l'autre s'avère une perte importante pour le développement forestier de la région. L'abolition de la CRÉO par le gouvernement québécois rend caduc les CRRNT, qui s'avéraient un lègue important au développement durable des territoires forestiers par l'adaptation des orientations aux réalités territoriales.

\section{NOTES}

${ }^{1}$ Beuret, Jean-Eudes. 2010. «De la négociation conflictuelle à la négociation concertative : un " point de passage transactionnel" ». Négociations, no 13, p. $43-60$.

${ }^{2}$ Leclerc, Edith et Caroline Andrew. 2013. «Les Tables de concertation de gestion intégrée des ressources forestières, est-ce que les GIRs gouvernent? ». In La gouvernance locale des forêts publiques québécoises. Une avenue de développement des régions périphériques?, sous la dir. de Guy Chiasson et Edith Leclerc, p. 127-146.

${ }^{3}$ Leclerc, Edith. 2013. «Construction des nouveaux territoires de la gouvernance forestière au Québec: Regards sur les régions du Bas-Saint-Laurent et de l'Outaouais ». Thèse de Doctorat, Gatineau, Département des sciences sociales, Université du Québec en Outaouais, 277 p.

${ }^{4} \mathrm{La}$ forêt outaouaise appartient au domaine bioclimatique de la forêt feuillue en zone tempérée nordique, alors que la plus grande proportion du territoire forestier québécois est en zone boréale.

${ }^{5} \mathrm{La}$ Direction générale régionale du ministère responsable de la forêt, la CRÉ et la CRRNT ont fait des démarches conjointes afin d'obtenir un BMMB dans la région. "La mission du BMMB est de rendre accessibles les bois sur le marché libre afin de favoriser l'utilisation optimale des ressources forestières dans le but de maximiser les revenus et la création de richesse découlant des activités d'aménagement forestier, et ce pour l'ensemble de la société québécoise. » (Bureau de mise en marché des bois, 2011) Le bureau de la division Sud-Ouest est situé à Maniwaki, en Outaouais. 